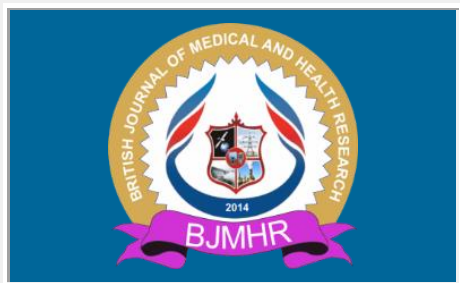

\title{
BJMHR
}

British Journal of Medical and Health Research

Journal home page: www.bjmhr.com

\section{Recurrent Pleural Effusion In Primary Systemic Amyloidosis - A Rare Manifestation Of A Rare Disease}

\author{
Sunil Kumar, Aarsh Shah*, Pradeep Sareddy, Archit Jain \\ Department of General Medicine, SBKS MIRC, Sumandeep Vidyapeeth, Waghodia, \\ Piparia, Vadodara - 391760
}

\begin{abstract}
Amyloidosis is a clinical disorder caused by extracellular and/or intracellular deposition of insoluble abnormal amyloid fibrils that alter the normal function of the tissues. Their clinical presentations consists of peripheral neuropathy, hepatomegaly, nephrotic syndrome, restrictive cardiomyopathy, purpura and other skin manifestations, bleeding diathesis and pleural effusion. Kidneys are the most common organs affected by primary amyloidosis. They present with swelling over ankles, feet, legs, fatigue, shortness of breath, weight loss, numbness in hand and feet but rarely present with unilateral significant pleural effusion. Similarly, we report a 58 years old male who presented with generalized weakness and fatiguability, lower limb edema and shortness of breath on exertion. Patient after being diagnosed as renal amyloidosis, was discharged and on follow up presented with unilateral left sided significant pleural effusion.
\end{abstract}

Keywords: Renal Amyloidosis, Recurrent pleural effusion, Primary systemic amyloidosis 


\section{INTRODUCTION}

Amyloidosis is the term used to refer to the extracellular and/or intracellular tissue deposition of fibrils composed of low molecular weight subunits of a variety of proteins. These deposits may result in a wide range of clinical manifestations depending upon their location, type and the amount of deposition.

These subunit proteins forming amyloid deposits are derived from soluble precursors which have undergone conformational changes that lead to the adoption of a predominantly antiparallel beta-pleated sheet configuration. Multiple different human protein precursors of amyloid fibrils are known. Amyloid has a characteristic gross pathologic and microscopic appearance, showing apple-green birefringence with polarized light microscopy of Congo red stained tissue.

Amyloidosis had been classified based on the site of deposition and presence or absence of other diseases. ${ }^{[1]}$ The term "Generalized" or "Systemic" has been used to describe deposition in multiple anatomic sites and "localized" used to describe deposition in one anatomic site. The term "secondary" is used to describe patients with coexisting disease like multiple myeloma and "primary" for patients with no such coexistent disease. ${ }^{[2]}$

\section{CASE REPORT}

A 58 years old male, presented to the outpatient department of our hospital with chief complaints of breathlessness on less than routine work, generalized weakness and bilateral lower limb swelling. Patient had no complaint of chest pain, orthopnoea, paroxysmal nocturnal dyspnoea. Patient was vitally stable and jugular venous pressure was not raised. On examination, fine crepitations were present bilaterally over the chest. Rest systems were normal on examination.

On being investigated, patient had haemoglobin of 12.4 , total counts -6400 , platelets -3.09 lakh, creatinine - 1.1, ESR - 26, LFT were within normal limits. Patient had 2+ albumin in urine. Patient's ECG was grossly normal. Patient's BNP level was done which was 1126 (higher than normal range). Chest x-ray revealed minimal bilateral blunting of costophrenic angles suggestive of bilateral minimal pleural effusion which was not very significant. Ultrasonography of abdomen and pelvis was within normal limits with normal sized kidneys. All these signs, symptoms and investigations indicated the diagnosis of heart failure initially. Patient was put on antibiotics, diuretics, anti-platelets and other symptomatic management. However, his 2D-echo revealed no regional wall abnormality or valvular disorder. His only significant finding was severe concentric LVH. His ejection fraction was normal (55\%). Hence, the diagnosis of heart failure leading to albuminuria and pedal edema was excluded. Further, patient's 24 hours urinary protein was done which was $2134 \mathrm{mg}$. Serum albumin of the patient 
was only $2.3 \mathrm{~g} / \mathrm{dL}$. Patient did not have any complaint of hematuria, reduced urine output yet. Nephrology opinion was taken. ANA was negative. Anti-PLA2r antibody of the patient was also negative -0.07 (normal range is less than 0.7). Following this, Patient's renal biopsy and abdominal fat pad biopsy was done. Three fat strands were taken and sent for the examination. The apple green birefringence was seen on congo red stained smear by polarized microscopy suggestive of amyloid deposits on both the organ biopsies. Patient was given injection pulse steroid therapy with methyl prednisolone and injection cyclophosphamide (as per its infusion protocol). Patient was discharged on maintenance oral steroids, diuretics, tab. Soda bicarbonate and multivitamins. Patient was explained about the complications of the disease, progression of the disease and the need for renal dialysis in future. Patient was advised to administer cyclophosphamide on follow up visits at fixed intervals but the patient did not comply as he felt better symptomatically than before. Patient was now a diagnosed case of renal amyloidosis. After about 3 months, patient presented again with similar symptoms but with more in intensity. This time with worsening urinary complaints of decreased urine output, progressive pedal edema, breathlessness on minimal exertion. Patient was admitted and his chest x-ray revealed left sided moderate pleural effusion. On HRCT thorax, patient had moderate to gross pleural effusion with underlying lung collapse of left lower lobe. Patient gave the history of pleurocentesis at a local hospital near his locality before 15 days where he had gone for his check up in the view of his symptoms and pleural fluid being removed due to presence of pleural effusion on the left side. It was recurrent pleural effusion. Creatinine on admission was 4.2 and it was in rising trend. Urine routine had 4+ albumin and 24 hours urinary protein was $5158 \mathrm{mg}$. His urine output was less than $500 \mathrm{ml}$ in 24 hours. Patient was put on haemodialysis on alternate day. 2D ECHO was done which had similar findings as before but with grade II diastolic dysfunction. Cardiology advice was taken where the expert suggested it might be cardiac involvement due to amyloidosis which is typical of concentric LVH without significant ECG changes. However, shining myocardium and signs of restrictive cardiomyopathy which is typical of cardiac amyloidosis was not visualized. Approx. $500 \mathrm{ml}$ pleural fluid was tapped. Fluid was transudative in nature. Pleural fluid analysis was total cells - 250 (lymphocytes $75 \%$, polymorphs $-15 \%$, mesothelial cells - 10\%), ADA - 13, LDH - 141, sugar - 106 against corresponding sugar of 122 , protein - 0.6, albumin - 0.2. It was negative for acid fast bacilli, CBNAAT and no growth on culture. During the stay of another 5 days, he again developed left sided pleural effusion where again another approximately $300 \mathrm{ml}$ was tapped. Pleural effusion was resistant to diuretic therapy and persisted even after haemodialysis. Intercostal drainage tube was placed on the left side in the view of recurrent pleural effusion. Patient was advised pleural biopsy to rule out pleural amyloidosis which is a common cause of pleural effusion. But patient and relatives were not willing for the same. Prognosis was explained to the patient's 
relatives. Patient took discharge against medical advice. Patient was prescribed steroids, diuretics, oral soda bicarbonate, antibiotics, iron supplements, multi-vitamins and maintenance haemodialysis with intercostal drainage tube and dialysis line catheter in situ. Patient was assumed to be having primary systemic amyloidosis with renal and lung involvement and/or cardiac involvement. Later patient died due to complications of the disease.

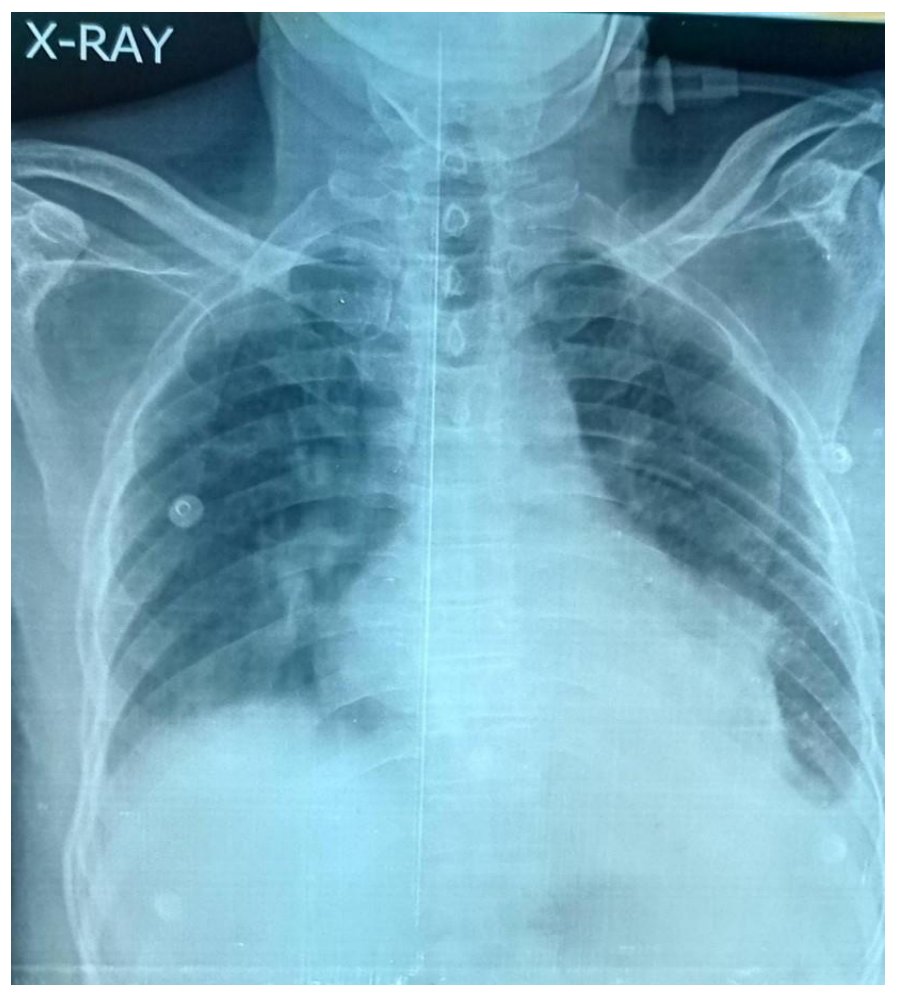

Figure 1: Chest $x$ ray showing bilateral minimal to mild pleural effusion during first visit.

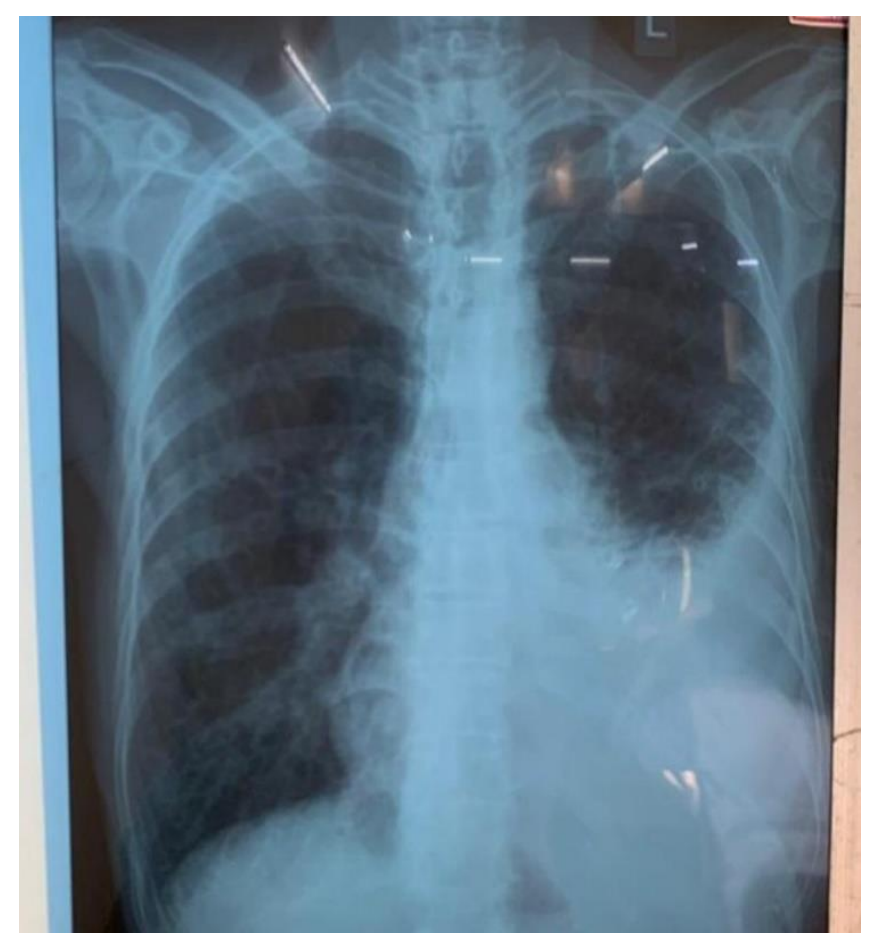

Figure 2: Chest $x$ ray showing left sided massive pleural effusion on subsequent visit. 


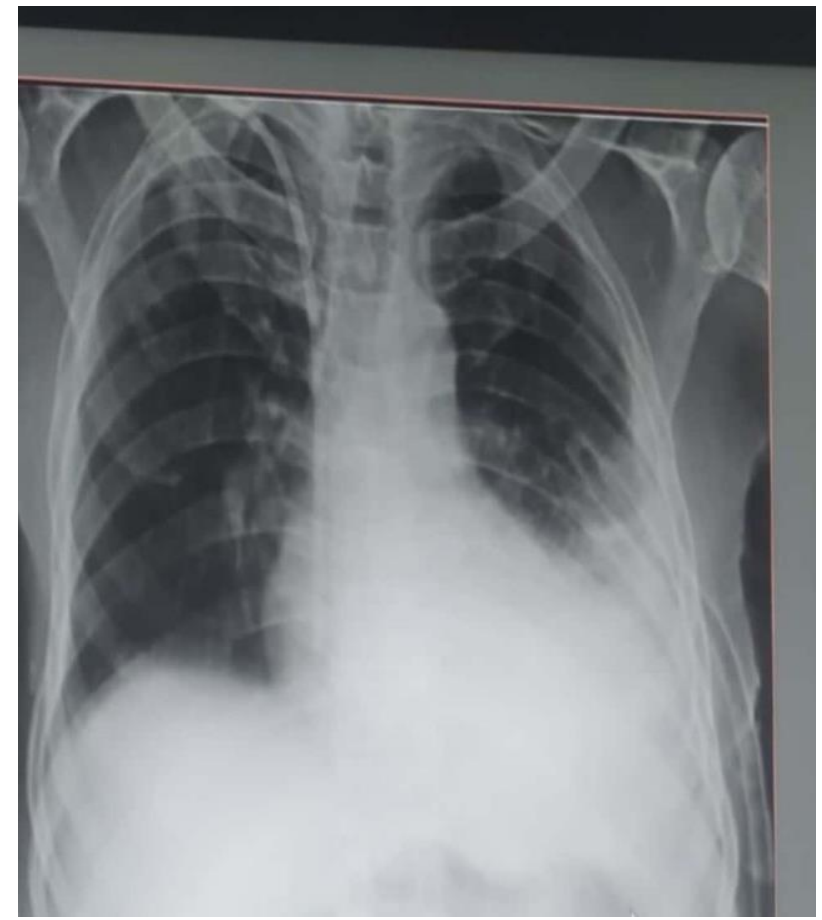

Figure 3: Chest $x$ ray showing pleural effusion with insertion of ICD after recurrent pleural effusion with DLC line catheter in situ.

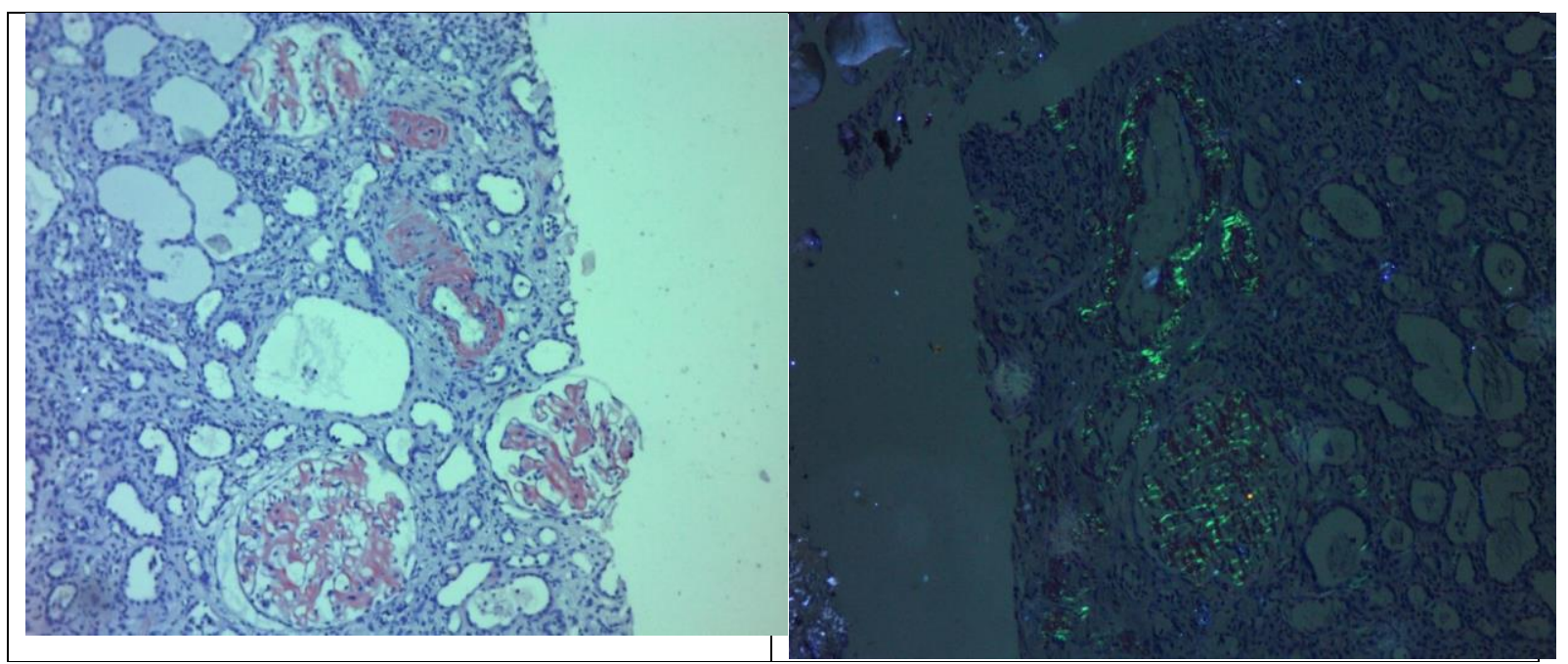

Figure 4a and $4 b$ - renal biopsy images of the patient with congo red staining in light microscopy and polarizing microscopy showing amyloid deposits.

\section{DISCUSSION}

Amyloidosis is a very rare disorder. The principal types of amyloidosis are the AL (primary) and AA (secondary) types, although other types of amyloid (eg, ATTR) are clinically important, some of which are common and others rare. AL amyloidosis is due to deposition of protein derived from immunoglobulin light chain fragments. It is a complication of plasma cell dyscrasia. AL amyloidosis is a systemic disorder that can present with a variety of signs and symptoms, including heavy proteinuria (usually in the nephrotic range) and edema, hepatosplenomegaly, unexplained heart failure, and the carpal tunnel syndrome. ${ }^{[3]}$ Although virtually all patients have multisystem amyloid deposition, it is common to present with 
evidence of mainly one organ being affected. As we saw in our case, the patient initially presented with unexplained signs and symptoms and later presented with pleural effusion proving involvement of only one organ which initially was kidney. Organ-specific amyloid is the one where amyloid deposition can be isolated to a single organ, such as the skin, eye, heart, pancreas, or genitourinary tract, resulting in specific syndromes.

Renal involvement most often presents as asymptomatic proteinuria or clinically apparent nephrotic syndrome ${ }^{[4]}$ Cardiac involvement can lead to diastolic or, usually later in the diseases course, systolic dysfunction and the symptoms of heart failure. ${ }^{[5]}$ Pulmonary manifestations of amyloidosis include persistent pleural effusions, parenchymal nodules (amyloidomas), tracheobronchial infiltration, and rarely pulmonary hypertension. ${ }^{[6,7,8]}$ Other manifestations include gastrointestinal disease, musculoskeletal disease, neurological abnormalities, skin disorders and others. Biopsy remains the mainstay of diagnosis of amyloidosis. Biopsies can be obtained from clinically uninvolved sites, like subcutaneous fat, minor salivary glands, rectal mucosa or from dysfunctional organs (eg, kidney, nerve). Biopsy of subcutaneous fat with Congo red staining and examination using polarizing microscopy has an overall sensitivity of 57 to 85 percent and a specificity of 92 to 100 percent for primary (AL) or secondary (AA) amyloidosis. ${ }^{[9]}$ The diagnostic sensitivity is higher with multiorgan involvement. From this, we could infer that our patient was having multi-system involvement who had positive fat pad biopsy report. Fat pad aspiration or biopsy has a low sensitivity for amyloidosis in patients with a single involved organ. Some patients have a predisposing underlying disorder before the presentation with amyloid, while others present with no such history but with an amyloid manifestation such as otherwise unexplained heart failure or nephrotic syndrome which happened in our case. In some cases, immunohistochemistry can be used to identify the type of protein subunit. ${ }^{[10]}$ This is most reliable for AA and transthyretin (TTR) amyloid and is less so for AL amyloid.

Therapy is aimed at the underlying plasma cell dyscrasia in primary (AL) amyloidosis. Previously patients were treated with combination of prednisone, melphalan and colchicine. ${ }^{[11]}$ Cyclophosphamide, Thalidomide, Dexamethasone appears to be a highly effective initial chemotherapy regimen for the treatment of systemic AL amyloidosis according to a study conducted by Ashutosh D. Wechalekar et al in 2006. ${ }^{[12]}$ The most common cause of the respiratory amyloid disease is secondary to systemic AL Amyloidosis which accounts for up to $80 \%$ of pulmonary amyloid ${ }^{[13]}$. Moreover, it has been reported that $88 \%$ of patients with systemic amyloid have pulmonary disease. ${ }^{[14]}$ Previously a similar case has been reported by Sunny George et al 'primary systemic amyloidosis: a rare cause of pleural effusion.' in 2014. ${ }^{\text {[15] }}$ A study published in Hindawi in 2015, the authors observed a case of recurrent pleural effusion in primary pulmonary amyloidosis. Here the pleural biopsy was positive for the deposition of 
amyloidosis. ${ }^{[16]}$ Amyloidosis AL with severe renal and cardiac involvement: a very rare association of terrible prognosis, two case reports were reported by M. Dolores RedondoPachón et al in 2012. ${ }^{[17]}$

\section{CONCLUSION}

Thus, few cases have been reported around the world mimicking our case. Amyloidosis is a rare disease and it can present with rarer manifestations that would directly not lead to its diagnosis. However, newer modalities are under progress for its diagnosis and newer treatments are being developed for the same to ease the patients suffering from the disease. Clinicians must keep in mind this type of atypical presentation of renal amyloidosis presenting with pleural effusion and progressing to primary systemic amyloidosis. Clinicians must consider and recognise the patients presenting with amyloidosis and keep in mind the progression of the disease and its fatal complications.

\section{REFERENCE}

1. Thompson PJ, Citron KM. Amyloid and the lower respiratory tract. Thorax Feb 1983;38(2):84e7.

2. Utz JP, Swensen SJ, Gertz MA. Pulmonary amyloidosis. The Mayo Clinic Experience from 1980 to 1993. Ann Intern Med Feb 15, 1996;124(4):407e13.

3. Merlini G, Dispenzieri A, Sanchorawala V, et al. Systemic immunoglobulin light chain amyloidosis. Nat Rev Dis Primers 2018; 4:38.

4. Kurita N, Kotera N, Ishimoto Y, et al. AA amyloid nephropathy with predominant vascular deposition in Crohn's disease. Clin Nephrol 2013; 79:229.

5. Bhogal S, Ladia V, Sitwala P, et al. Cardiac Amyloidosis: An Updated Review with Emphasis on Diagnosis and Future Directions. Curr Probl Cardiol 2018; 43:10.

6. Ross P Jr, Magro CM. Clonal light chain restricted primary intrapulmonary nodular amyloidosis. Ann Thorac Surg 2005; 80:344.

7. Ikeda S, Takabayashi Y, Maejima Y, et al. Nodular lung disease with five year survival and unilateral pleural effusion in AL amyloidosis. Amyloid 1999; 6:292.

8. BoydKing A, Sharma O, Stevenson K. Localized interstitial pulmonary amyloid: a case report and review of the literature. Curr Opin Pulm Med 2009; 15:517.

9. Duston MA, Skinner M, Meenan RF, Cohen AS. Sensitivity, specificity, and predictive value of abdominal fat aspiration for the diagnosis of amyloidosis. Arthritis Rheum $1989 ; 32: 82$.

10. Arbustini E, Morbini P, Verga L, Merlini G. Light and electron microscopy immunohistochemical characterization of amyloid deposits. Amyloid 1997; 4:157. 
11. Benson M. Treatment of AL amyloidosis with melphalan, prednisone, and colchicine. Arthritis and rheumatism, Vol. 29, No. 5 (May 1986).

12. Adams D, Gonzalez-Duarte A, O'Riordan WD, et al. Patisiran, an RNAi Therapeutic, for Hereditary Transthyretin Amyloidosis. N Engl J Med 2018; 379:11.

13. Benson MD, Waddington-Cruz M, Berk JL, et al. Inotersen Treatment for Patients with Hereditary Transthyretin Amyloidosis. N Engl J Med 2018; 379:22.

14. Maurer MS, Schwartz JH, Gundapaneni B, et al. Tafamidis Treatment for Patients with Transthyretin Amyloid Cardiomyopathy. N Engl J Med 2018; 379:1007.

15. Berk JL, Suhr OB, Obici L, et al. Repurposing diflunisal for familial amyloid polyneuropathy: a randomized clinical trial. JAMA 2013; 310:2658.

16. Lauren Tada, Humayun Anjum, W. Kenneth Linville, Salim Surani. "Recurrent Pleural Effusions Occurring in Association with Primary Pulmonary Amyloidosis." Hindawi Publishing Corporation Case Reports in Pulmonology Volume 2015, Article ID 421201, 5 pages.

17. M. Dolores Redondo-Pachón, Ricardo Enríquez, Ana E. Sirvent, Encarna Andrada, Raimundo García-Del Moral, Isabel Millán, Francisco Amorós. “Amyloidosis AL with severe renal and cardiac involvement: a very rare association of terrible prognosis, two case reports". Nefrologia 2012;32(6):848-50.

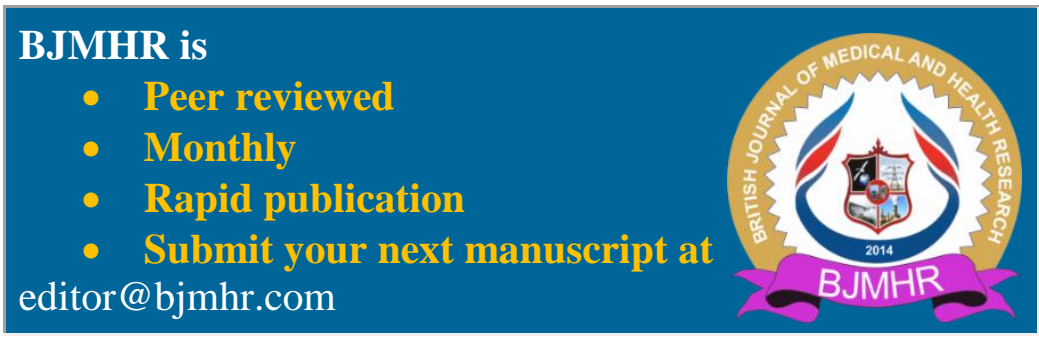

